\author{
Change in Negative Attention Bias Mediates the Association Between \\ Attention Bias Modification Training and Depression Symptom Improvement \\ Christopher G. Beevers ${ }^{1}$, Kean J. Hsu², David M. Schnyer ${ }^{1}$, Jasper A.J. Smits ${ }^{1}$, and Jason \\ Shumake $^{1}$ \\ ${ }^{1}$ Department of Psychology and Institute for Mental Health Research, University of Texas \\ at Austin \\ ${ }^{2}$ Georgetown University Medical Center
}

\begin{abstract}
Author Note
(C) 2021, American Psychological Association. This paper is not the copy of record and may not exactly replicate the final, authoritative version of the article. Please do not copy or cite without authors' permission. The final article will be available, upon publication, via its DOI: $10.1037 /$ ccp0000683. This project was supported in part by funding from the National Institute of Mental Health (NIHM, R33MH109600). The content is solely the responsibility of the authors and does not necessarily represent the official views of the National Institutes of Health. Analysis code for this article is available in our dataverse (https://doi.org/10.18738/T8/G2KV5B) and the data are available via NIMH's data archive (http://dx.doi.org/10.15154/1522538). Address correspondence to Christopher Beevers, University of Texas at Austin, Institute for Mental Health Research, 305 E. 23rd St., E9000, Austin, TX 78712; email: beevers@utexas.edu. Christopher G. Beevers (iD https://orcid.org/0000-0002-4480-0902
\end{abstract}




\begin{abstract}
Objective: Attention bias modification training (ABMT) is purported to reduce depression by targeting and modifying an attentional bias for sadness-related stimuli. However, few tests of this hypothesis have been completed. Method: The current study examined whether change in attentional bias mediated a previously reported association between ABMT condition (active ABMT, sham ABMT, assessments only; $N=145$ ) and depression symptom change among depressed adults. The pre-registered, primary measure of attention bias was a discretized eye tracking metric that quantified the proportion of trials where gaze time was greater for sad stimuli than neutral stimuli. Results: Contemporaneous longitudinal simplex mediation indicated that change in attentional bias early in treatment partially mediated the effect of ABMT on depression symptoms. Specificity analyses indicated that in contrast to the eye-tracking mediator, reaction time assessments of attentional bias for sad stimuli (mean bias and trial level variability) and lapses in sustained attention did not mediate the association between ABMT and depression change. Results also suggested that mediation effects were limited to a degree by suboptimal measurement of attentional bias for sad stimuli. Conclusion: When effective, ABMT may improve depression in part by reducing an attentional bias for sad stimuli, particularly early on during ABMT.

Public Health Statement: Prior work has shown that ABMT reduces depression but it is unclear why this is so. The current study confirms that ABMT works by reducing a tendency to focus attention on negative information, which in turn reduces symptoms of depression.
\end{abstract}

Keywords: clinical trial, mediation, attention bias modification, structural equation models 


\section{Change in Negative Attention Bias Mediates the Association Between Attention Bias Modification Training and Depression Symptom Improvement}

Depression is a common, recurrent, and impairing condition that predicts future suicide attempts, interpersonal problems, unemployment, substance abuse, and other negative outcomes (Kessler et al., 2012, Wells, 1992). Cognitive theory (Beck and Haigh, 2014; Disner et al., 2011) asserts that negatively biased attention (among other cognitive biases) has an important role in the maintenance of the disorder. That is, depressed individuals selectively attend to negative information and have difficulty disengaging attention from negative stimuli. These attentional biases, in turn, reinforce a sad mood and contribute to a persistent depressive episode.

A great deal of correlational research supports the idea that depressed individuals' attention is negatively biased (Gotlib and Joormann, 2010). A meta-analysis found that depressed participants have a stronger attention bias toward negative stimuli than non-depressed participants, particularly when assessed with a dot-probe task $(k=12, n=$ 937, $d=0.52, p<.001$; Peckham et al., 2010). Similarly, two meta-analyses of eye tracking studies found that depression was associated with sustained maintenance of gaze on dysphoric stimuli (Armstrong and Olatunji, 2012, Suslow et al., 2020). Further, among adults with elevated depression severity, greater negative attention bias was associated with symptom worsening over the subsequent five weeks ( $r=-.42$; Disner et al., 2017). Importantly, using eye tracking assessments, negatively biased attention predicted future

increases in depression symptom severity (Beevers et al., 2011) and prolonged mood persistence among people with MDD (Clasen et al., 2013; Sanchez et al., 2013).

Given this correlational evidence, it follows that Attention Bias Modification Training (ABMT) could be used to target and reduce attentional biases for sad information and improve depression symptoms. However, relatively few rigorous clinical trials of ABMT have been completed with depressed adults (Fodor et al., 2019; Mogoaşe et al., 2014). In a clinical trial with college students reporting mild to severe symptoms of 
depression $(N=77)$, active ABMT was associated with significant reductions in depressive symptoms at post-training $(d=1.38,95 \%$ CI $[0.78,1.97]$ for active vs. sham ABMT; $d=$ 1.45, $95 \%$ CI $[0.83,2.08]$ for active ABMT vs. assessment only) compared to sham ABMT and assessment only. These group differences were maintained at a 3 month follow-up (Yang et al., 2015). A larger study $(N=145)$ compared the efficacy of active versus sham ABMT to an assessment only comparison condition among adults with depression and at least a moderate negative attentional bias (Hsu et al., 2021). In that study, active ABMT reduced gaze bias for sad stimuli and improved self-reported and interviewer-rated depression symptoms to a greater extent than assessment only $(d \mathrm{~s}=-.57,-.49)$ and sham $\operatorname{ABMT}(d \mathrm{~s}=-.41,-.42)$. Change in depression symptoms did not differ between sham ABMT and assessment only.

Given these treatment efficacy findings, the current study examines putative mediators of active ABMT in the previously reported clinical trial (Hsu et al., 2021). Here, we focus on the ABMT treatment target - attentional bias for sad information measured with eye gaze - as a putative mediator. Prior work showed that active ABMT for depression led to greater reductions in negative attentional bias than sham ABM; however, formal mediation was not examined (Beevers et al., 2015). Other work found that change in negative attention bias did not mediate the association between ABMT and depression, but it did mediate the association between ABMT and rumination (Yang et al., 2015).

In the current study, we test whether attentional bias for sad stimuli mediates the association between ABMT and change in depression symptoms using longitudinal mediation analyses (Goldsmith et al., 2018, Preacher, 2015). An ideal design for longitudinal mediation in the context of a clinical trial involves the repeated assessment of the mediator and outcome at the same assessment time points. This allows for the use of various types of longitudinal structural equation mediation models that take advantage of the temporal information from the repeated assessments (5 weekly assessments in the current study) to quantify the direct effects of treatment on the mediator and outcome, as 
well as the indirect effect of treatment on the outcome through a mediator.

Models with either contemporaneous (e.g., negative attention bias at week 1 predicts depression at week 1) or lagged (e.g., negative attention bias at week 1 predicts depression at week 2) associations can be fit to clinical trial data where the mediator and outcome have been assessed repeatedly. Contemporaneous mediation effects typically yield better fitting models in clinical trials because the lag between measurements is often too long to observe the mediator change before the outcome (Goldsmith et al., 2018).

We hypothesized that negative attention bias would mediate the association between active ABMT (vs assessment only) and depression symptom improvement. However, we did not have hypotheses with respect to differences between contemporaneous and lagged (weekly) effects; thus, we examined both models in the analyses. Further, in addition to the pre-specified mediator - attentional bias for sad stimuli measured with eye tracking - we explored mediator specificity by examining whether mean bias for sad stimuli, attention bias variability (Lazarov et al., 2018), and lapses in sustained attention (Dinges et al., 1997) also serve as mediators. Finally, although the primary comparison treatment was the assessment only condition, we also examined whether negative attention bias mediated efficacy when sham ABMT was the comparison condition.

\section{Methods}

\section{Overview of Randomized Clinical Trial Procedures and Prior Findings}

Detailed descriptions of the randomized clinical trial have been published elsewhere (Hsu et al., 2018 and Hsu et al., 2021). Briefly, participants in this single center, randomized, blinded clinical trial (NCT 02880215) were 145 adults 18 to 40 years old recruited from the Austin, TX, USA community through social media advertisements, flyering, and community postings among other approaches. Participants were eligible if they could provide informed consent, were fluent in English, scored 13 or greater on the Quick Inventory of Depressive Symptoms - Self-Report (QIDS-SR) at the baseline assessment, and had greater total fixation time for negative than neutral stimuli on at least 
$37.5 \%$ of trials of an eye-tracking task (see Assessments below for explanation of why this criterion was chosen). Participants were excluded if they reported suicidal behavior or significant active suicidal ideation within the past six months assessed with the Columbia-Suicide Severity Rating Scale (C-SSRS; Posner et al., 2011), if they met criteria for current or past bipolar or psychotic disorders or for substance use disorders of mild or greater severity within the past 12 months on the Mini International Neuropsychiatric Interview (MINI; Lecrubier et al., 1998), or if they were taking current opioid analgesics or systemic corticosteroid use. Individuals on antidepressant medication were required to have had no changes in medication and dosage in the past 12 weeks. See supplemental materials for additional details about participant recruitment.

Treatment conditions included active ABMT, sham ABMT, and assessments only. The active ABMT condition used the dot-probe task to putatively train attention away from dysphoric stimuli (sad faces and negative scenes) and toward neutral stimuli by altering the probe contingency so it appeared in the location of the neutral stimuli $80 \%$ of the time. The sham ABMT condition used the same task and stimuli but was intended to assess attention bias rather than modify it as the probe had an equal probability of appearing in the location of the neutral and dysphoric stimuli. The assessment only condition did not complete any attention training but instead completed the depression and attention bias assessments. Participants were scheduled to complete 20 minute ABMT sessions twice a week in the clinic and 6 minute ABMT sessions three times per week at home for four weeks. More information about the treatment conditions, randomization, and dosage is provided in the study protocol (Hsu et al., 2018) and the supplemental materials.

General instructions were provided before randomization and were intentionally vague about the purpose and design of ABMT. For example, in the consent form, the purpose of the study was described as, "You have been asked to participate in a research study about the development of an attention bias modification training (ABMT) intervention designed to target and reduce negative attention bias and thereby reduce 
symptoms of depression. The purpose of this study is to better understand the role of negative attention bias in depression, and how specifically targeting and reducing negative attention bias can affect depressive symptoms." Participants were then told they would be randomized to one of three conditions, two of which involved completing twice per week in-clinic ABMT and three times per week at home ABMT. All participants were told that repeated practice with this task might improve their symptoms of depression.

As previously reported (Hsu et al., 2021), intent-to-treat analyses of the time $\mathrm{x}$ group interaction indicated that active ABMT reduced depression symptom severity on the primary outcome, the QIDS-SR, to a greater extent than the other conditions. Compared to assessment only, depression was reduced by an additional $0.62 \pm 0.23(p=.008, d=$ -0.57) points per week. Compared to sham ABMT, depression was reduced by an additional $0.44 \pm 0.24$ QIDS-SR $(p=.067, d=-0.41)$ points per week. Sham ABMT and assessment only did not significantly differ in terms of symptom change $(p=.41, d=$ -0.17). Further, the time $\mathrm{x}$ group interaction indicated that active ABMT led to a reduction in the odds of a trial where gaze was greater for sad than neutral stimuli relative to assessment only ( $\mathrm{OR}=0.9, p=.066)$ and sham ABMT $(\mathrm{OR}=0.91, p=.075)$. On the other hand, the odds of eye gaze bias toward sad stimuli for sham ABMT remained equivalent to assessment only $(\mathrm{OR}=1, p=.97)$. This initial evidence of ABMT efficacy and potential target engagement prompted us to examine whether negative attentional bias mediates this observed ABMT treatment effect.

\section{Sample size justification}

Of the 145 participants randomized to an intervention, 48 were allocated to active ABMT, 49 were allocated to sham ABMT, and 48 were allocated to assessments only. This mediation analysis is a secondary aim of the clinical trial and sample size requirements were determined by the primary aim of detecting a mean difference in efficacy (Hsu et al., 2018), not estimating a mediation effect. However, the expected power of this analysis to detect key effects is an important metric for evaluating the limitations of the reported 
results. Therefore, we conducted 1000 simulations of randomly generated data sets fixed to the same sample size as our real data but drawn from a "true" contemporaneous simplex model or linear growth model in which we fixed model coefficients to our theoretical priors: namely, a ground truth assumption that the overall indirect effect of active ABMT on bias would partially mediate $(\mathrm{d}=0.25)$ a total medium-sized $(\mathrm{d}=0.5)$ effect of active ABMT on the final depression observation. These simulations showed that the unconstrained and constrained contemporaneous simplex models were able to detect a significant mediation effect in $62.2 \%$ and $78.3 \%$ of the simulations, respectively. Thus, for the limited goal of rejecting the null hypothesis of no mediation effect, these analyses were reasonably powered (although we acknowledge that the unconstrained simplex model might have benefited from a larger sample).

\section{Assessments}

Data from all available participants were utilized in the mediation analyses $(N=$ 145). Depression symptom severity and negative attention bias were obtained at baseline and then weekly for four weeks (i.e., week $0,1,2,3,4)$ for a total of five assessments.

Depression. The pre-registered primary outcome was the self-report version of the 16-item Quick Inventory of Depression Symptoms (QIDS-SR). The QIDS-SR assesses the nine DSM-IV symptom domains for depression and has been shown to be highly reliable, internally consistent, and sensitive to changes in symptom severity (Rush et al., 2003; Trivedi et al., 2004). The internal consistency for the QIDS-SR in the current sample was quite low at baseline (Cronbach's $\alpha=.23$ ), likely due to a truncated distribution associated with inclusion criteria, but acceptable in subsequent assessments (Cronbach's $\alpha$ ranged from .70 to .78).

Dot-probe. During the dot-probe task, eye movements were recorded at a rate of $500 \mathrm{~Hz}$ using a video-based eye tracker (Eye-Link 1000 Plus Desktop Mount; SR Research, Osgoode, Ontario, Canada). Before the presentation of practice trials, participants completed a 13-point calibration routine in order to map eye position to screen coordinates. 
The calibration process was then validated with a 13-point retest routine. The calibration process was accepted if there was less than a 0.5 degree visual angle between initial calibration and subsequent validation. After the initial calibration process, quality of eye tracking was maintained through drift checks between each trial. Participants were required to maintain their gaze on the fixation cross before the next set of stimuli was presented; if fixation was not detected, a single-point drift correction procedure was initiated.

\section{Measurement of negative attention bias}

Psychometric issues have been raised about traditional methods of scoring the

behavioral reaction time data obtained from the dot-probe task (Rodebaugh et al., 2016). As a result, we carefully operationalized inclusion criteria and ABMT mediator metrics, both of which involve the dot-probe task. As outlined in our protocol paper for this ABMT trial (Hsu et al., 2018), we examined the one-week stability of several dot-probe eye-tracking metrics to identify a suitable attention bias criterion. Analyses from an independent sample of 85 adults indicated that the number of trials where total fixation time was greater for sad stimuli than neutral stimuli had the best one-week stability $(r=$ 0.67) out of several dot-probe metrics of negative attention bias.

Further, as a criterion of sufficient bias for study eligibility, spontaneous drift between sufficient and insufficient bias across a 1-week period was minimized if a participant had at least $37.5 \%$ of trials with total fixation time greater for sad than neutral stimuli. Thus, in order to be eligible to participate in the study, participants had to have at least 36 out of 96 trials where gaze towards sad stimuli was greater than gaze towards neutral stimuli.

For the ABMT mediation analyses, we scaled the number of trials where fixation time for sad stimuli was greater than fixation time for neutral stimuli as a proportion of the number of valid trials at each assessment. Split-half internal consistency for the dot-probe task at baseline was adequate (Spearman-Brown corrected $r=0.42,95 \%$ CI $[0.27,0.54])$ for the number of trials where net fixation time was greater for negative than 
neutral stimuli. This metric also had adequate one-week stability $(r=.54)$ in the assessment-only condition from baseline to week 1 .

\section{Exploratory ABMT mediators}

Reaction-time metrics of attentional bias and variability. Attentional bias using reaction time data was operationalized consistent with standard conventions (Mogg et al., 2008) using an R package developed in our lab called itrak https://github.com/jashu/itrak. Prior to creating bias scores, we first excluded incorrect responses, responses that were faster than $200 \mathrm{~ms}$, longer than $1500 \mathrm{~ms}$, or were 3 median absolute deviations beyond the individual's median. Using these standard cut-offs, $9.5 \%$ of the trials were identified as invalid $(8.6 \%, 10 \%$, and $9.9 \%$ for the active, sham, and assessment-only groups, respectively).

There are two types of trials on the dot-probe task that are central to quantifying attention bias: congruent and incongruent trials. For congruent trials, the negative stimulus and probe are presented in the same location. For instance, if the negative stimulus is presented on the left, then the probe $(\mathrm{O}$ or $\mathrm{Q})$ is also presented on the left. For incongruent trials, the probe and negative stimulus are in opposite locations. For instance, if the negative stimulus is presented on the left, then the probe $(\mathrm{O}$ or $\mathrm{Q})$ is presented on

the right. A person with a tendency to attend to negative stimuli should be faster to identify the probe location for congruent trials compared to incongruent trials, as the latter requires shifting of attention to the opposite side of the visual field.

Zvielli et al. (2016) proposed that attention bias is dynamic and fluctuates over time, so using overall mean reaction time to congruent and incongruent trials may miss important information. Rather than using bias scores that are averaged across all trials, they developed trial-level bias scores (TLBS) that create difference scores between congruent and incongruent trials that are within close temporal proximity to each other. In our modified approach to calculating TLBS, the reaction time of each trial is differenced from the weighted mean reaction time of all trials of opposite type, with closer trials 
weighted more heavily than more distant trials (as a function of the inverse square of trial distance). In other words, each congruent trial is subtracted from the weighted (according to trial proximity) mean of all incongruent trials (rather than just the most proximal out of a maximum of 5 trials as in Zvielli et al., 2016), and the weighted mean of all congruent trials is subtracted from each incongruent trial (see supplemental materials for more detail). Difference scores that are greater than 0 indicate a bias towards sad stimuli, whereas bias scores that are less than 0 indicate a bias away from sad stimuli. Thus, this method creates a time series of bias scores throughout the course of the task and putatively captures dynamic changes in attention bias.

The TLBS mean bias and variability scores were obtained by calculating the mean and standard deviation, respectively, of each TLBS time series. Positive mean bias scores are interpreted as an attention bias directed towards negative stimuli, whereas negative scores indicate a bias away from negative stimuli. Split-half reliability for mean attentional bias was good, Spearman-Brown corrected $r=0.84,95 \%$ CI $[0.79,0.88]$, but this metric had poor one-week stability in the assessment-only group from baseline to week 1 ( $r=$ .12). Split-half reliability was excellent for attentional bias variability, Spearman-Brown corrected $r=0.96,95 \%$ CI [0.94, 0.97], and one-week stability in the assessment only group was adequate $(r=.57)$. One week stability was examined in the assessment only group as they did not receive any intervention designed to modify an attentional bias (which could artificially deflate the stability of attentional bias).

Sustained attention. A computerized reaction time test called the Psychomotor Vigilance Task (PVT; Dinges et al., 1997) measured sustained attention. The PVT is a high-signal load reaction time test that has been employed in hundreds of studies to measure attentional deficits because it is reliable, valid, and highly sensitive to changes in sustained attention (for a review, see Lim and Dinges, 2008). Participants attended to a fixation cross at the center of a computer screen. At random intervals, a millisecond timer appeared at the screen center (2 to 10 second inter-trial intervals). Participants were 
instructed to press a button the instant they detected the timer. The button press stopped the timer and displayed the reaction time for 1 second. The PVT was 10 minutes in length and required constant attention in order to detect the timer. The primary assessment of sustained attention using the PVT was number of attentional lapses (responses greater than $500 \mathrm{~ms})$. This metric also had excellent one-week stability $(r=.90)$ in the assessment only condition from baseline to week 1. As a secondary PVT metric, we also examined the mean of the fastest 10 percent of the trials. This metric also had excellent one-week stability $(r=.70)$ in the assessment only condition from baseline to week 1.1

\section{Data Analyses}

A structural equation model (SEM) of longitudinal mediation, specifically a three-group dual process simplex model (Goldsmith et al., 2018), was fit to the raw data using full-information maximum likelihood as implemented by the OpenMx package in $\mathrm{R}$ (Neale et al., 2016). Primary mediation models used the proportion of valid trials where gaze bias was greater than $50 \%$ for sad versus neutral stimuli as our measure of attention bias for sad stimuli, and QIDS-SR was the depression symptom outcome. Models with either contemporaneous (e.g., negative bias at Week 1 predicts depression at Week 1) or lagged (e.g., negative bias at Week 1 predicts depression at Week 2) mediation paths were fit to the data. A latent growth model was also examined to determine whether mediation was observed using a different longitudinal mediation model.

These models were decomposed into indirect/mediated effects (all paths that go from training condition to the final depression measure through any measure of attention bias) and direct effects (all paths that go from training condition to the final depression

\footnotetext{
${ }^{1}$ We did not report split-half metrics for the PVT because, by design, attentional lapses should be uncommon events, the probability of which is greatly increased as a function of trial number. Therefore, while we can still assess the between-session stability of the count of attentional lapses, we do not calculate split-half reliability because within session it is, by design, unstable and temporally dependent, with identical stimuli across trials.
} 
measure through any prior measure of depression but not through any measure of attention bias). These effects are defined as the products of each chain of path coefficients that lead to the final outcome. Given that there were 3 experimental groups, there are two treatment contrasts for every effect: one for active vs. no training and one for sham vs. no training. Each time-specific effect is summed to obtain either an overall indirect effect or an overall direct effect, with the overall indirect effect being of primary interest for testing the significance of a mediation effect. Because normal-theory precision estimates perform poorly for coefficient products, it is recommended that CIs be obtained by a bias-corrected bootstrap (Hayes, 2017), which makes no assumptions about the shape of the sampling distribution of coefficients or their products. Following the example of Goldsmith et al., 2018, we used 1000 bootstrap replications to calculate 95\% CIs.

Simplex mediation model specification. Unless otherwise noted, model specification follows the Simplex Models for Mediation as outlined by Goldsmith et al. (2018). In a simplex model, each occasion of observed measurement for both the mediator and outcome indicates a "true" latent factor. With only a single indicator for each latent variable, Goldsmith et al. (2018) suggest setting all factor loadings equal to 1 so that the model is identifiable. Goldsmith et al. (2018) suggest estimating occasion-specific AR1 coefficients - that is, allow the magnitude of the autoregressive effects to change over time. Given that the duration of our study (4 weeks) and lag between measurement occasions (1 week) are comparably short, we expect any week-to-week fluctuations in the AR1 effects to reflect sampling noise. Therefore, the AR1 paths for each measurement were constrained to be equal across time. Moreover, since we are allowing variances to differ at baseline (because of distortions in the baseline distributions caused by eligibility screening), some additional constraint must be made to the model in order for it to remain identifiable.

The mediator and outcome measures are joined through $b$ paths between the latent variables. In the contemporaneous model the $b$ paths are contemporaneous and in the lagged model the $b$ paths are lagged by 1 week. For both lagged and contemporaneous $b$ 
paths, the $b$ paths are constrained to be equal over time and across treatments, which offers more parsimony and precise estimation (Goldsmith et al., 2018). This enforces the theoretical prior that the relationship between the attentional bias mediator and the depression outcome should be reasonably consistent over time and independent of the type of training received. In other words, we do not consider it plausible to allow the causal relationship between attentional bias and depression to change in sign or magnitude depending on when or which training was received.

The $a$ paths are those between the treatment group dummy variables and the latent bias mediator at each time point. The $c$ ' paths are the "direct" effects between the treatment dummy group variables and the latent depression outcome at each time point. They represent any residual influences of training on depression not explained by changes in bias. This could include, for example, a placebo effect or non-specific effects due to habituation to the emotional stimuli. Given that sham training, by design, should not modify attention bias, the argument could be made that its $a$ paths should be fixed to 0; any effect it has on depression must be achieved through its $c^{\prime}$ paths. By design, only active training should be able to exert an indirect effect through the ab paths. We directly test this possibility by comparing a model that allows sham training to influence attention bias against one that does not. The model that specifies these theoretical constraints on sham training effects will be preferred unless a likelihood ratio test indicates that removing them will significantly improve model fit.

We depart from Goldsmith et al. (2018) in that we did not set the paths between treatment contrasts and the final measures of bias and depression to 0 . This is because in our design the final time point represented the end of treatment, not a post-treatment followup. Although technically treatment was not ongoing at the final assessment, the time elapsed since the final training session was too short to rule out a direct effect of training on the final measurements. Therefore, occasion-specific paths between training contrast variables and all post-baseline measurements of attention bias and depression were freely 
estimated.

The residual variances of observed QIDS and bias scores are set to be equal over time for the post-baseline measurements, but are freely estimated for the baseline measurements. The reason for this is that this sample was selected to exclude participants with low negative attention bias and low levels of depression; thus, we expect the variance at baseline to be less than subsequent time points because of this truncation of values. Variances of the dummy variables and baseline latent variables are freely estimated. All variances are constrained to positive values. The covariance between mediator and outcome at baseline is modeled in order to adjust for any initial cross-sectional association of mediator and outcome when estimating the b paths. Covariance is also estimated between the dummy variables defining the training contrasts. Each post-baseline factor includes an occasion-specific disturbance term, which represents all omitted causes plus any random or measurement error. This is constrained to have a lower bound of 0 .

Rescaling of QIDS-SR and attentional bias scores. Given that the scales of raw QIDS-SR and attentional bias scores differ by an order of magnitude, some form of standardization is necessary. In the primary analyses of outcomes (Hsu et al., 2021), we calculated effect sizes based on the pooled standard deviation observed at the final time point of measurement. By using the same divisor to scale the measurements here, the units of the subsequent effects decomposition analysis can likewise be interpreted as a Cohen's $d$ measure of treatment differences at the end of the study. We also centered the standardized variables to the mean of group means across all occasions, but this decision is arbitrary and has no impact on the results of this analysis.

Model fit metrics. We report four model fit indices for each model: $\chi^{2}$, root mean square of error of Approximation (RMSEA), Akaike's information criterion (AIC), and the Bayesian information criterion (BIC). The $\chi^{2}$ value compares the observed covariance matrix with the theoretical covariance matrix proposed by the model. A significant $(\mathrm{p}<.05)$ difference may indicate poor fit, though our primary criterion of 
acceptable fit was an RMSEA 90\% CI with a lower bound less than 0.05 and an upper bound less than 0.10. RMSEA estimates the difference between the examined model and a hypothetical model where every component in the model is related to every other component. A significant $(\mathrm{p}<.05)$ difference would mean that we should reject the model. An RMSEA of 0 would be considered ideal and any value less than 0.05 would be considered a good fit. AIC and BIC are model fit indices that reflect a penalty for the number of parameters in the model (with BIC using a larger penalty), and are reported to facilitate comparisons between models with this tradeoff in mind. For all fit indices, the smaller the value of the statistic, the better the fit.

Model selection justification. As recommended by Goldsmith et al. (2018) in their tutorial on applying longitudinal structural equation mediation models to clinical trial data, fitting competing plausible alternative models allows "for a sensitivity analysis of mediation estimates that span an often ignored domain of uncertainty-selecting the correct model structure." The latent growth model is specifically important to consider because it models change as occurring within individuals according to a theoretically constrained function (in this case linear slope). If the form of change is in truth approximately linear, then this should lead to more accurate and reliable coefficient estimates. For example, when we simulated a fully mediated effect of linear change in depression as a function of linear change in bias using an asymptotically large sample size, this full-mediation effect (d $=-0.5)$ could only be recovered by the latent growth model; the simplex model estimated the total effect correctly but, without the ability to capture the within-individual trajectories, only estimated a partial mediation effect $(d=-0.08)$.

\section{Results}

\section{Simplex Mediation Models with Primary Attentional Bias Mediator}

\section{Contemporaneous model}

The pre-registered, primary mediator was the proportion of trials where eye gaze was greater for sad stimuli than neutral stimuli based on eye-tracking fixation data. In this 
contemporaneous mediation model, ABMT indirectly influenced depression symptoms through its effect on attentional bias for sad stimuli at the same time point. The overall fit of this model was considered acceptable: $\chi^{2}=52, \mathrm{df}=41, \mathrm{p}=0.12$; RMSEA $[90 \% \mathrm{CI}]=$ $0.043[0,0.075], \mathrm{p}=0.6 ; \mathrm{AIC}=3459 ; \mathrm{BIC}=3398$.

We then removed the paths from sham ABMT to attentional bias, as sham ABMT is not theoretically expected to influence negative attentional bias. This constrained model also had an acceptable fit, $\chi^{2}=53.9, \mathrm{df}=45, \mathrm{p}=0.17$; RMSEA $[90 \% \mathrm{CI}]=0.037[0$, 0.069], $\mathrm{p}=0.71 ; \mathrm{AIC}=3443 ; \mathrm{BIC}=3392$, suggesting that sham ABMT did not substantively alter negative attentional bias. Model fits for the unconstrained and constrained models also did not differ, LRT $\chi^{2}=1.9, \mathrm{df}=4, p=0.76$. Further, all fit statistics (RMSEA, AIC, and BIC) are superior for the more constrained model. This suggests that there is no reason to reject the more parsimonious and theoretically plausible model that indirect effects were only possible for active ABMT.

As can be seen in Figure 1 and Table 1, participants who received ABMT were estimated to show reductions in negative attentional bias at all time points except for week 2 (i.e., $\mathrm{a}_{11}$ to $\mathrm{a}_{14}$ in Table 1 ). The strongest, and only statistically significant, effect of training on attentional bias was observed for the effect of active ABMT at week $1\left(\mathrm{a}_{11}=\right.$ $-0.396[-0.697,-0.099])$. Further, there was a significant effect of attentional bias on depression (path $b$ in Table 1). Participants who experienced a $1 S D$ reduction in bias were estimated to show a $0.244[0.123,0.444] S D$ decrease in depression at the same time point. There were also significant autoregressive effects for both the depression assessments (y) and the attentional bias assessments $(\mathrm{m})$, suggesting stability in these measurements over time. 
Tests of the indirect effects (a total of 10 different indirect effects of ABMT -> bias -> depression) for the constrained contemporaneous model are presented in Table 2. The overall indirect effect is the sum of all coefficient products for each unique path from training condition to final depression that travels through at least one bias measurement. Based on 1,000 bootstrap samples, a bias-corrected $95 \%$ confidence interval for the total indirect effect of active ABMT was entirely below zero, $-0.204[-0.407,-0.039]$. Change in attentional bias for sad stimuli significantly mediated the effect of active ABMT on depression symptom improvement.

There was also evidence that active ABMT training influenced depression independent of its effect on attentional bias, with an overall direct effect of $-0.44[-0.774$, -0.092]. The overall direct effect of sham ABMT [95\% CI] was -0.29 [-0.62, -0.032]. Thus, active ABMT had both an indirect and direct effect on depression, for a total effect [95\% CI] of $-0.644[-0.924,-0.327]$. This total effect was a little more than twice as large as the total effect of sham ABMT on depression.

It is interesting to note from the decomposition of different temporal pathways (Table 2) that all significant indirect effects included a path from active ABMT to the week 1 negative attentional bias measurement, which was then propagated through autoregressive effects to later time points (e.g., active ABMT -> bias1 -> bias2 -> bias3 -> dep3 -> dep4). This implies that, within the active ABMT condition, the mediation effects on final depression were driven primarily by early changes in negative attentional bias.

See supplemental materials for a full reporting of the initial unconstrained contemporaneous model and models with other constraints. Importantly, these additional models arrive at similar conclusions to the models presented here.

\section{1-week lag model}

In addition to a contemporaneous model, we also examined lagged mediation where attentional bias for sad stimuli predicted depression one week later. The initial 1-week lagged mediation model places no constraints on training effects. It provides an adequate 
fit to the data, $\chi^{2}=59.9, \mathrm{df}=41, \mathrm{p}=0.028, \operatorname{RMSEA}(90 \% \mathrm{CI})=0.056[0.019,0.086], \mathrm{p}$ $=0.34, A I C=3466, B I C=3406$.

This 1-week lagged mediation model indicates that there is a significant association between bias at time $\mathrm{t}-1$ and depression at time t, $\mathrm{b}=0.164[0.029,0.358]$. There is also a significant indirect effect of active ABMT on depression via 1-week lagged bias (-0.102 $[-0.304,-0.007])$, although this effect is somewhat smaller than what was observed in the contemporaneous models. Given the weaker evidence for lagged mediation (effect sizes were approximately half as large and only borderline significant) compared to the contemporaneous models, we refer the reader to the supplemental materials for more information regarding lagged mediation.

\section{Simplex Mediation Models with Secondary Attentional Bias Mediators}

These models were identical to the contemporaneous simplex model used in the primary analysis except the secondary attentional bias mediators were substituted for the primary eye-tracking based mediator. Any other differences are also noted.

\section{Mean Attention Bias for Sad Stimuli Measured with Reaction Time (RT)}

The variance of mean behavioral reaction time attentional bias was not freely estimated at baseline but rather constrained to equal the variance at all subsequent occasions. Unlike the count of trials with gaze-fixation bias, this measure was not used to screen for eligibility, and consequently variance at baseline was not artificially compressed relative to subsequent time points.

Using traditional mean reaction time as the attentional bias mediator, the fit of this model was poor, $\chi^{2}=82.2, \mathrm{df}=42, \mathrm{p}<.001, \operatorname{RMSEA}[90 \% \mathrm{CI}]=0.081[0.055,0.11], \mathrm{p}=$ $0.028, \mathrm{AIC}=3672, \mathrm{BIC}=3614$. In addition, compared to the eye gaze mediator, the autoregressive effect of the mean reaction time attentional bias was notably smaller (.48 vs 0.70) with a larger standard error. The disturbance term for the mean attentional bias scores was much larger for all occasions of measurement, except the final one, with larger standard errors at all occasions. Therefore, the reliability and validity of the effects 
decomposition and mediation tests based on this model are more suspect. Given this, we present these effects in the supplemental materials and will not discuss them further. Given this poor initial fit, we also did not examine any constrained models.

\section{Attention bias variability measured with RT}

Using attention bias variability measured with reaction time as as the attentional bias mediator also does not provide a strong model fit, $\chi^{2}=84$, $\mathrm{df}=42, \mathrm{p}<.001$, RMSEA $[90 \% \mathrm{CI}]=0.083[0.057,0.11], \mathrm{p}=0.021, \mathrm{AIC}=3377, \mathrm{BIC}=3319$. However, in contrast to mean attention bias, bias variability appeared to be highly reliable-in fact, it had similar reliability as the depression measure in terms of having relatively large autoregressive coefficients (0.825) and small disturbances and residual errors (see supplemental materials). However, there was no evidence for an effect of attentional bias variability on depression, as the estimated b path was very close to zero, 0.019 [-0.028, 0.069]. Similarly, there was a near-zero indirect effect for both active ABM, -0.017 [-0.079, 0.038], and sham ABM, $-0.011[-0.065,0.017]$. Nearly all of the total effects of active and sham ABM on final depression score were estimated to be independent of attentional bias variability (see supplemental materials for more detail).

\section{Sustained attention measured with Psychomotor Vigilance Task (PVT)}

Sustained attention was examined as an alternative (or competing) mediator for the effect of ABMT on depression improvement. Since the baseline PVT assessment was not used to determine study eligibility, there is no rationale to allow for residual variance of PVT measures to differ at baseline. Instead, all residual errors are constrained to equality within each repeated measure.

Model fit indices for mediation by sustained attention were acceptable and highly similar for attention lapses, $\chi^{2}=62.4, \mathrm{df}=42, \mathrm{p}=0.022 ;$ RMSEA $[90 \% \mathrm{CI}]=0.058$ $[0.023,0.087], \mathrm{p}=0.31 ; \mathrm{AIC}=2941 ; \mathrm{BIC}=2883$ and reaction times, $\chi^{2}=74.1, \mathrm{df}=42, \mathrm{p}$ $=0.0016 ;$ RMSEA $[90 \% \mathrm{CI}]=0.073[0.044,0.099], \mathrm{p}=0.088 ; \mathrm{AIC}=2915 ; \mathrm{BIC}=2857$. For both PVT measures, sustained attention appeared to be a highly stable trait (AR1 = 
0.96-1) that is not modified by ABMT. Not surprisingly, overall indirect effects of ABMT training were very close to 0 , suggesting that sustained attention did not mediate the effect of ABMT on depression symptoms (see supplemental materials for more detail).

If these PVT mediation models had acceptable fit statistics, we would have followed with a parallel mediation model. That is, a model that includes both the attentional bias and PVT mediators to examine whether each mediator explained unique variance in the outcome. However, these PVT models did not meet this criterion, so parallel mediation was not necessary.

\section{Simplex Mediation Model Comparing Only Active and Sham ABMT}

This pre-registered study was designed to compare active ABMT and sham ABMT to an assessment only condition. Thus, the assessment only condition was used as the comparison in all of the analyses to this point. However, an important question to examine is whether negative attentional bias mediates the effect of active ABMT on depression compared to sham ABMT. Thus, this analysis was the same as the primary contemporaneous simplex mediation analysis except participants in the assessment only condition were removed from the model and active ABMT vs sham ABMT was directly compared.

The fit statistics for this model were excellent: $\chi^{2}=37.4$, df $=39, \mathrm{p}=0.54$; RMSEA $[90 \% \mathrm{CI}]=0[0,0.066], \mathrm{p}=0.85 ; \mathrm{AIC}=2162 ; \mathrm{BIC}=2089$. There was a significant difference between active and sham ABMT on bias scores at week 1, with active ABMT associated with a significant reduction in attentional bias compared to sham $\operatorname{ABMT}\left(a_{1}=-0.381[-0.713,-0.003]\right)$. The contemporaneous influence of attentional bias on depression was also significant $(b=0.349[0.202,0.606])$, with a 1 SD decrease in bias predicting .35 SD decrease in depression. Further, all indirect effects on final depression that stemmed from active ABMT effects on week-1 bias were significant compared to sham ABMT. All indirect effects stemming from later effects on attentional bias (weeks 2 - 4) were not significant. 
However, despite the point estimate being almost identical to the overall indirect effect of active ABMT relative to assessment-only, the confidence interval was wider and not entirely below zero for the overall indirect effect of active relative to sham ABMT $(-0.222[-0.499,0.013])$. Thus, partial mediation effects contrasting active and sham ABMT were similar to the mediation effects contrasting active ABMT to the assessment-only condition, albeit with more uncertainty, likely due to decreased sample size from removing the assessment-only participants from the model.

\section{Latent Growth Model with Primary Attentional Bias Mediator}

To determine whether the observed mediation effects were sensitive to the simplex models, we next considered latent growth as an alternative mediation model. This model examined whether ABMT assignment (active and sham ABMT versus control) predicts the linear rate of change in attentional bias (measured with eye gaze), which in turn predicts the linear rate of change in depression.

The latent growth model provided a good fit for the data, $\chi^{2}=72.4$, df $=53, \mathrm{p}=$ 0.04; RMSEA [90\% CI $]=0.05[0.012,0.077], \mathrm{p}=0.47 ; \mathrm{AIC}=3430 ; \mathrm{BIC}=3396$. Consistent with the simplex models, we noted that the effect of sham ABMT on change in attentional bias was estimated to be near 0 . Thus, we then fit a constrained model where the effect of sham ABMT on attention bias was set to 0 . This constrained model also provided a good fit for the data, $\chi^{2}=72.6, \mathrm{df}=54, \mathrm{p}=0.047$; RMSEA $[90 \% \mathrm{CI}]=0.049$ $[0.0062,0.076], \mathrm{p}=0.51 ; \mathrm{AIC}=3426 ; \mathrm{BIC}=3395$, suggesting that sham ABMT had a minimal effect on change in negative attentional bias. Model fits for the constrained and unconstrained models did not differ, LRT $\chi^{2}=0.2$, df $=1, p=0.68$. The constrained model was retained.

We placed one additional constraint on this constrained model to improve model performance. We constrained the direct effects of sham and active ABMT to have a negative sign (i.e., impose an upper bound of 0 ). This enforces an assumption that, while the direct effect of training can be 0 , it is implausible that the direct effect of either ABMT 
condition could increase depression.

This constraint lead to more plausible estimates, as a model without this constraint found a large negative indirect effect (i.e., active ABMT decreased depression) that was compensated by a modest positive direct effect (i.e., active ABMT increased depression). Model fit for this bounded model was acceptable, $\chi^{2}=72.6, \mathrm{df}=54, \mathrm{p}=0.047$; RMSEA $[90 \% \mathrm{CI}]=0.049[0.0063,0.076], \mathrm{p}=0.51 ; \mathrm{AIC}=3426 ; \mathrm{BIC}=3395$. This model is described further, as it is most parsimonious and has the most plausible estimates. (All three versions of the growth models can be inspected in supplemental materials and none would lead to substantively different conclusions.)

This bounded model estimated that change in attention bias for sad stimuli was significantly associated with change in depression: for every 1 SD change in attention bias slope, an almost $2 \mathrm{SD}$ change in depression was expected, $\mathrm{b}=1.898[0.409,5.433]$ (see Table 3). Active ABMT was also associated with a reduction in attention bias over time, $\mathrm{b}$ $=-0.073[-0.156,-0.006]$. Recall that the effect of sham ABMT on attentional bias was set to be 0 .

The tests for mediation are presented in Table 4. The indirect effect of active ABMT on depression change through change in bias slope was estimated to be [95\% CI] $-0.554[-0.929,-0.174]$. The direct effects $[95 \% \mathrm{CI}]$ of active ABMT was $0[-0.239,0]$ and sham ABMT was -0.229 [-0.544, 0]; both contained 0 in their respective $95 \%$ CIs. Thus, the total effect of active ABMT on change in depression was entirely explained by the impact of active ABMT on change in attentional bias. There was a modest direct effect of sham ABMT on depression change, but this was not mediated by attention bias (sham ABMT had no effect on negative attentional bias and was set to 0). Thus, compared to assessment only, active ABMT appears to decrease depression by reducing a negative attentional bias.

\section{Discussion}

This study examined mediation effects for a prior trial that reported depression symptom reductions associated with completion of active ABMT compared to assessment 
only (Hsu et al., 2021). The current study found that reductions in attention for sad stimuli (when measured with eye tracking) partially accounted for the beneficial effect of ABMT on depression symptoms. This was observed using simplex longitudinal mediation that modeled effects between the weekly assessments of the mediator and outcome and in latent growth models where linear change in bias mediated the association between ABMT and depression improvement.

Mediation results in the simplex models were strongest for contemporaneous mediation-that is, attentional bias for sad stimuli was more strongly associated with change in depression symptom severity at the same time point rather than one week later. The results also suggest that change in attentional bias happens early in ABM, whereas depression symptom change occurs later on during treatment. This accounts for why the bias-mediated effect was smaller than the direct effect of ABMT on depression change-early change had to propagate through more intermediate paths. Finally, reaction time measures of attention bias for sad stimuli (mean bias and attention bias variability) and lapses in sustained attention did not mediate the effects of ABM, suggesting mediation specificity for attentional bias for sad stimuli.

This is among the first evidence to suggest that ABMT for depression, when effective, appears to improve depression symptoms in part via its impact on attentional bias for sad stimuli. This is consistent with work in social anxiety, which has also documented that change in attentional biases for threat stimuli mediate the effect of ABMT on change in anxiety symptoms (e.g., Kuckertz et al., 2014). It is notable that mediation was only observed when attentional bias for sad stimuli was measured with eye gaze. Eye gaze methods have the potential to be a more sensitive measure of attention bias, as they more directly measure line of gaze compared to behavioral measures where attention often has to be inferred from differences in reaction times to stimuli. However, eye gaze is not immune to psychometric challenges depending on the paradigm within which they are used (Price et al., 2019). Further, eye gaze provides a large number of 
possible metrics (e.g., mean gaze time, number of fixations, latency to first fixation), which increases analytical flexibility and the potential for Type I error.

To combat this problem, we previously collected data from an independent sample to determine which eye gaze metric might optimally be associated with depression and have reasonable stability over time (see Hsu et al., 2018)). This was particularly important because consistency in the ranking of individuals over time is crucial for studying individual differences (Hedge et al., 2018) and we also wanted to reliably identify depressed individuals with at least a moderate level of attention bias as part of the study eligibility criteria (Hsu et al., 2018). The eye tracking metric with the best psychometric properties (and the one used in the primary mediation analyses) was the proportion of trials where gaze time was greater for sad stimuli than neutral stimuli. Many prior studies have used total fixation time or a difference score between sad stimuli and neutral stimuli. Although there is nothing inherently wrong with using a difference score to create a bias metric, difference scores tend to be unreliable when the values subtracted from each other are highly correlated (Rodebaugh et al., 2016, Trafimow, 2015). This is the case with most reaction time and eye gaze metrics-for instance, mean reaction time to sad stimuli tends to be highly correlated with mean reaction time to neutral stimuli. Thus, we selected an eye gaze metric that did not depend on difference scores.

That said, there is certainly room to improve on the measurement of attention bias for sad stimuli even when using eye tracking methods. It was notable that in the simplex mediation models the disturbances (error) for the measurement of attention bias were about twice as large as the disturbances for self-reported depression. Week-to-week stability was also relatively modest. The attention variability trial level bias score (Zvielli et al., 2016) and lapses in sustained attention measured with the PVT did have small disturbances, suggesting that related constructs can be measured with high stability and low error. However, these metrics did not mediate the effect of ABMT on depression change.

One way to potentially improve the measurement of attention bias for sad stimuli is 
to use multiple assessments of attentional bias. Multiple assessments would allow for a latent mediation approach which could allow for modeling more signal to noise (Zhang and Yang, 2020). In addition to eye tracking with a dot-probe task that uses a proportion of trials where gaze is greater for sad stimuli than neutral stimuli, other tasks such as a passive viewing task (Beevers et al., 2011) or attention capture (Arnell et al., 2007), could be added to an assessment battery. Given that these tasks would be assessed repeatedly during the clinical trial, the ideal battery of assessments would need to be relatively efficient. The dependent measures (depression symptoms) would also ideally be measured with more than one assessment, also further lengthening the study protocol. An alternative to multiple assessments may be to consider incorporating computational models of behavioral reaction time (e.g., drift diffusion modeling, Price et al., 2018) and cognitive neuroscience methods, such as event-related potentials of attention (Kappenman et al., 2015), that often exhibit superior reliability to behavioral indices. More research is needed to identify an optimal battery of tasks that measure attentional bias for sad stimuli.

More work is also needed to determine the optimal lag between the assessment of the mediator and the outcome (Dormann and Griffin, 2015). The current study used a typical approach of assessing the mediator and outcome on a weekly basis throughout the clinical trial and found stronger support for contemporaneous mediation than mediation with one week lags between assessments. However, this weekly assessment schedule was selected based on tradition/superstition instead of any strong theoretical or empirical understanding of how attentional biases for sad stimuli might maintain depression. Indeed, others have suggested that contemporaneous mediation is most likely to be observed in clinical trials (Goldsmith et al., 2018), as it is rare that constructs are so well defined or theoretical models are so well specified (Fried and Robinaugh, 2020) that we have a precise understanding of when to measure the mediators and outcomes. Future work, perhaps using intensive assessment schedules (e.g., repeated daily assessments) might be useful for determining the optimal assessment lags between attentional biases and symptoms of 
psychopathology.

More frequent assessments would also help pinpoint the precise timing of when ABMT is most likely to change the putative mediators. The current study found that active ABMT had the strongest impact on negative attentional bias at week 1. Subsequent effects were smaller, suggesting that the effects of ABMT may wane over time. Future work may want to consider novel ways to further enhance the efficacy of ABMT (Woolridge et al., 2020) or even adopting Sequential Multiple Assignment Randomized Trial (SMART; Lei et al., 2012) study designs, where individuals can be randomized to different treatments once the salutary effects of ABMT begin to diminish. Interventions that target different cognitive biases (e.g., interpretation, memory) may be viable next-step treatments.

We also note that in the universe of potential mediators, we examined only a few potential candidate mediators. We focused primarily on measures of attention bias for sad stimuli, which we consider to be among the most obvious and theoretically relevant mediators (Disner et al., 2011). However, other cognitive factors might also be important to examine. Although the theoretical models are not well specified, candidate mediators of ABMT might include rumination (Yang et al., 2015), negative processing (Harmer et al., 2009), and cognitive control over emotional information (Quigley et al., 2020). Further, arguments can be made for examining non-cognitive mediators, such as improvements in sad mood and/or emotion regulation (Todd et al., 2012). In addition, resting state fMRI was collected as part of the study protocol for a subset of participants, allowing for future reports to examine change in correlated activity in neural circuits that support attentional bias for sad stimuli as potential mediators (Beevers et al., 2015). Given that attentional bias for sad stimuli partially mediated the effect of ABMT on depression and explained approximately $28 \%$ of the total effect in the contemporaneous simplex model, this suggests that other mechanisms may be important to consider.

It was also notable that mediation of ABMT effects on depression by attentional bias for sad stimuli (measured with eye tracking) was not specific to a particular statistical 
model, as mediation was also observed in two different models (i.e., simplex mediation and linear latent growth). These estimates of total effects are very similar to what we obtained from linear mixed effects models of change in QIDS-SR in the primary outcome paper (Hsu et al., 2021). This is perhaps not surprising given that these models also assume a linear rate of change. Nevertheless, it is encouraging that this different model specification, which was fit with full-information maximum likelihood incorporating additional covariates, converges on a similar conclusion about effect size as our initial analyses in the primary outcome paper. Notably, like the linear mixed effects models, the total effect size estimates are approximately twice as large for active ABMT vs. assessment only compared to sham ABMT vs. assessment only (between group $d \mathrm{~s}=-0.64$ vs.-0.29, respectively) and the active ABMT total effect is not within the 95\% CI of the sham ABMT total effect (see Table 2).

Important limitations include the recruitment of few participants with severe levels of depression (though $75 \%$ of the sample met criteria for current MDD) and suicidality, a focus on acute response to ABMT (the long-term effects of ABMT used in this trial remain unknown), a narrow age range (18 - 40 years old) in an effort to minimize the impact of cognitive aging on assessments, a higher than anticipated rate of attrition, and reliance on a WEIRD (white, educated, industrialized, rich, democratic) sample (Henrich et al., 2010). Further, at least a moderate attentional bias for sad stimuli was required for entry into the study, so results may not generalize to all participants with depression. Our pre-specified primary outcome was self-reported depression symptoms. Results may differ for interviewer-rated symptoms. Further, we examined depression symptom total scores, in line with the vast majority of past research in this area, but future work should consider examining the effects of ABMT on specific symptom profiles (Fried and Nesse, 2015, Kraft et al., 2019). Finally, we did not examine the generalizability of ABMT to other attentional bias tasks and power analyses suggested our sample size was adequate but less than ideal, especially in the case of the unconstrained simplex model.

In conclusion, findings suggest that reductions in attentional bias for sad stimuli 
(i.e., decreases in the number of trials where gaze time is greater for sad stimuli than neutral stimuli) partially account for reductions in self-reported depression associated with active ABMT compared to only completing assessments. This is consistent with cognitive models of depression which posit that negative attention bias contributes to the maintenance of depression and that changing such biases can lead to improvements in depression (Beck and Haigh, 2014; Disner et al., 2011). This work also suggests that ABMT has the potential to be a useful intervention for depressed adults with an attentional bias for sad stimuli. Future large scale clinical trials with a robust assessment schedule that allows for testing mediation will provide important information about the replicability of these effects and help to identify the active ingredients associated with ABMT. 


\section{References}

Armstrong, T., \& Olatunji, B. O. (2012). Eye tracking of attention in the affective disorders: A meta-analytic review and synthesis. Clin. Psychol. Rev., 32(8), $704-723$.

Arnell, K. M., Killman, K. V., \& Fijavz, D. (2007). Blinded by emotion: Target misses follow attention capture by arousing distractors in RSVP. Emotion, 7(3), 465-477.

Beck, A. T., \& Haigh, E. A. P. (2014). Advances in cognitive theory and therapy: The generic cognitive model. Annu. Rev. Clin. Psychol., 10, 1-24.

Beevers, C. G., Clasen, P. C., Enock, P. M., \& Schnyer, D. M. (2015). Attention bias modification for major depressive disorder: Effects on attention bias, resting state connectivity, and symptom change. J. Abnorm. Psychol., 124(3), 463-475.

Beevers, C. G., Lee, H.-J., Wells, T. T., Ellis, A. J., \& Telch, M. J. (2011). Association of predeployment gaze bias for emotion stimuli with later symptoms of PTSD and depression in soldiers deployed in iraq. Am. J. Psychiatry, 168(7), 735-741.

Clasen, P. C., Wells, T. T., Ellis, A. J., \& Beevers, C. G. (2013). Attentional biases and the persistence of sad mood in major depressive disorder. J. Abnorm. Psychol., 122(1), $74-85$.

Dinges, D. F., Pack, F., Williams, K., Gillen, K. A., Powell, J. W., Ott, G. E., Aptowicz, C., \& Pack, A. I. (1997). Cumulative sleepiness, mood disturbance, and psychomotor vigilance performance decrements during a week of sleep restricted to 4-5 hours per night. Sleep, $20(4), 267-277$.

Disner, S. G., Beevers, C. G., Haigh, E. A. P., \& Beck, A. T. (2011). Neural mechanisms of the cognitive model of depression. Nat. Rev. Neurosci., 12(8), 467-477.

Disner, S. G., Shumake, J. D., \& Beevers, C. G. (2017). Self-referential schemas and attentional bias predict severity and naturalistic course of depression symptoms. Cogn. Emot., $31(4), 632-644$. 
Dormann, C., \& Griffin, M. A. (2015). Optimal time lags in panel studies. Psychol. Methods, 20(4), 489-505.

Fodor, L. A., Georgescu, R., Cuijpers, P., Szamoskozi, S., David, D., Furukawa, T. A., \& Cristea, I. A. (2019). The effectiveness of cognitive bias modification interventions in anxiety and depressive disorders: A network Meta-Analysis.

Fried, E. I., \& Nesse, R. M. (2015). Depression sum-scores don't add up: Why analyzing specific depression symptoms is essential. BMC Med., 13, 72 .

Fried, E. I., \& Robinaugh, D. J. (2020). Systems all the way down: Embracing complexity in mental health research. BMC Med., 18(1), 205.

Goldsmith, K. A., MacKinnon, D. P., Chalder, T., White, P. D., Sharpe, M., \& Pickles, A. (2018). Tutorial: The practical application of longitudinal structural equation mediation models in clinical trials. Psychol. Methods, 23(2), 191-207.

Gotlib, I. H., \& Joormann, J. (2010). Cognition and depression: Current status and future directions. Annu. Rev. Clin. Psychol., 6, 285-312.

Harmer, C. J., O'Sullivan, U., Favaron, E., Massey-Chase, R., Ayres, R., Reinecke, A., Goodwin, G. M., \& Cowen, P. J. (2009). Effect of acute antidepressant administration on negative affective bias in depressed patients. Am. J. Psychiatry, $166(10), 1178-1184$.

Hayes, A. F. (2017). Introduction to mediation, moderation, and conditional process analysis, second edition: A Regression-Based approach. Guilford Publications.

Hedge, C., Powell, G., \& Sumner, P. (2018). The reliability paradox: Why robust cognitive tasks do not produce reliable individual differences. Behav. Res. Methods, 50(3), $1166-1186$.

Henrich, J., Heine, S. J., \& Norenzayan, A. (2010). Most people are not WEIRD. Hsu, K. J., Caffey, K., Pisner, D., Shumake, J., Risom, S., Ray, K. L., Smits, J. A. J., Schnyer, D. M., \& Beevers, C. G. (2018). Attentional bias modification treatment 
for depression: Study protocol for a randomized controlled trial. Contemp. Clin. Trials, 75, 59-66.

Hsu, K. J., Shumake, J., Caffey, K., Risom, S., Labrada, J., Smits, J. A. J., Schnyer, D. M., \& Beevers, C. G. (2021). Efficacy of attention bias modification training for depressed adults: A randomized clinical trial. Psychol. Med.

Kappenman, E. S., MacNamara, A., \& Proudfit, G. H. (2015). Electrocortical evidence for rapid allocation of attention to threat in the dot-probe task. Soc. Cogn. Affect. Neurosci., 10(4), 577-583.

Kessler, R. C., Avenevoli, S., Costello, E. J., Georgiades, K., Green, J. G., Gruber, M. J., He, J.-P., Koretz, D., McLaughlin, K. A., Petukhova, M., Sampson, N. A., Zaslavsky, A. M., \& Merikangas, K. R. (2012). Prevalence, persistence, and sociodemographic correlates of DSM-IV disorders in the national comorbidity survey replication adolescent supplement. Arch. Gen. Psychiatry, 69(4), 372-380.

Kraft, B., Jonassen, R., Heeren, A., Harmer, C., Stiles, T., \& Landrø, N. I. (2019). Attention bias modification in remitted depression is associated with increased interest and leads to reduced adverse impact of anxiety symptoms and negative cognition. Clin. Psychol. Sci., 7(3), 530-544.

Kuckertz, J. M., Gildebrant, E., Liliequist, B., Karlström, P., Väppling, C., Bodlund, O., Stenlund, T., Hofmann, S. G., Andersson, G., Amir, N., \& Carlbring, P. (2014). Moderation and mediation of the effect of attention training in social anxiety disorder. Behav. Res. Ther., 53, 30-40.

Lazarov, A., Suarez-Jimenez, B., Abend, R., Naim, R., Shvil, E., Helpman, L., Zhu, X., Papini, S., Duroski, A., Rom, R., Schneier, F. R., Pine, D. S., Bar-Haim, Y., \& Neria, Y. (2018). Bias-contingent attention bias modification and attention control training in treatment of PTSD: A randomized control trial. Psychol. Med., 1-9.

Lecrubier, Y., Sheehan, D., Hergueta, T., \& Weiller, E. (1998). The mini international neuropsychiatric interview. 
Lei, H., Nahum-Shani, I., Lynch, K., Oslin, D., \& Murphy, S. A. (2012). A "SMART" design for building individualized treatment sequences. Annu. Rev. Clin. Psychol., $8,21-48$.

Lim, J., \& Dinges, D. F. (2008). Sleep deprivation and vigilant attention. Ann. N. Y. Acad. Sci., 1129, 305-322.

Mogg, K., Holmes, A., Garner, M., \& Bradley, B. P. (2008). Effects of threat cues on attentional shifting, disengagement and response slowing in anxious individuals. Behav. Res. Ther., 46(5), 656-667.

Mogoaşe, C., David, D., \& Koster, E. H. W. (2014). Clinical efficacy of attentional bias modification procedures: An updated meta-analysis. J. Clin. Psychol., 70(12), $1133-1157$.

Neale, M. C., Hunter, M. D., Pritikin, J. N., Zahery, M., Brick, T. R., Kirkpatrick, R. M., Estabrook, R., Bates, T. C., Maes, H. H., \& Boker, S. M. (2016). OpenMx 2.0: Extended structural equation and statistical modeling. Psychometrika, 81(2), 535-549. https://doi.org/10.1007/s11336-014-9435-8

Peckham, A. D., McHugh, R. K., \& Otto, M. W. (2010). A meta-analysis of the magnitude of biased attention in depression. Depress. Anxiety, 27(12), 1135-1142.

Posner, K., Brown, G. K., Stanley, B., Brent, D. A., Yershova, K. V., Oquendo, M. A., Currier, G. W., Melvin, G. A., Greenhill, L., Shen, S., \& John Mann, J. (2011). The Columbia-Suicide severity rating scale: Initial validity and internal consistency findings from three multisite studies with adolescents and adults.

Preacher, K. J. (2015). Advances in mediation analysis: A survey and synthesis of new developments. Annu. Rev. Psychol., 66, 825-852.

Price, R. B., Brown, V., \& Siegle, G. J. (2018). Computational modeling applied to the Dot-Probe task yields improved reliability and mechanistic insights. Biol. Psychiatry. 
Price, R. B., Woody, M. L., Panny, B., \& Siegle, G. J. (2019). Pinpointing mechanisms of a mechanistic treatment: Dissociable roles for overt and covert attentional processes in acute and Long-Term outcomes following Attention-Bias modification. Clin. Psychol. Sci., 2167702619842556.

Quigley, L., Wen, A., \& Dobson, K. S. (2020). Cognitive control over emotional information in current and remitted depression. Behav. Res. Ther., 132, 103658.

Rodebaugh, T. L., Scullin, R. B., Langer, J. K., Dixon, D. J., Huppert, J. D., Bernstein, A., Zvielli, A., \& Lenze, E. J. (2016). Unreliability as a threat to understanding psychopathology: The cautionary tale of attentional bias. J. Abnorm. Psychol., 125(6), 840-851.

Rush, A. J., Trivedi, M. H., Ibrahim, H. M., Carmody, T. J., Arnow, B., Klein, D. N., Markowitz, J. C., Ninan, P. T., Kornstein, S., Manber, R., Thase, M. E., Kocsis, J. H., \& Keller, M. B. (2003). The 16-item quick inventory of depressive symptomatology (QIDS), clinician rating (QIDS-C), and self-report (QIDS-SR): A psychometric evaluation in patients with chronic major depression. Biol. Psychiatry, $54(5), 573-583$.

Sanchez, A., Vazquez, C., Marker, C., LeMoult, J., \& Joormann, J. (2013). Attentional disengagement predicts stress recovery in depression: An eye-tracking study. $J$. Abnorm. Psychol., 122(2), 303-313.

Suslow, T., Hußlack, A., Kersting, A., \& Bodenschatz, C. M. (2020). Attentional biases to emotional information in clinical depression: A systematic and meta-analytic review of eye tracking findings. J. Affect. Disord.

Todd, R. M., Cunningham, W. A., Anderson, A. K., \& Thompson, E. (2012). Affect-biased attention as emotion regulation. Trends Cogn. Sci., 16(7), 365-372.

Trafimow, D. (2015). A defense against the alleged unreliability of difference scores. Cogent Mathematics, 2(1), 1064626. 
Trivedi, M. H., Rush, A. J., Ibrahim, H. M., Carmody, T. J., Biggs, M. M., Suppes, T., Crismon, M. L., Shores-Wilson, K., Toprac, M. G., Dennehy, E. B., et al. (2004). The inventory of depressive symptomatology, clinician rating (IDS-C) and Self-Report (IDS-SR), and the quick inventory of depressive symptomatology, clinician rating (QIDS-C) and Self-Report (QIDS-SR) in public sector patients with mood disorders: A psychometric evaluation. Psychol. Med., 34(1), 73-82.

Wells, K. B. (1992). The course of depression in adult outpatients.

Woolridge, S. M., Harrison, G. W., Best, M. W., \& Bowie, C. R. (2020). Attention bias modification in depression: A randomized trial using a novel, reward-based, eye-tracking approach. J. Behav. Ther. Exp. Psychiatry, 71, 101621.

Yang, W., Ding, Z., Dai, T., Peng, F., \& Zhang, J. X. (2015). Attention bias modification training in individuals with depressive symptoms: A randomized controlled trial. $J$. Behav. Ther. Exp. Psychiatry, 49(Pt A), 101-111.

Zhang, Q., \& Yang, Y. (2020). Autoregressive mediation models using composite scores and latent variables: Comparisons and recommendations. Psychol. Methods, 25(4), 472-495.

Zvielli, A., Vrijsen, J. N., Koster, E. H. W., \& Bernstein, A. (2016). Attentional bias temporal dynamics in remitted depression. J. Abnorm. Psychol., 125(6), 768-776. 


\section{Table 1}

SEM paths for constrained contemporaneous mediation model

\begin{tabular}{lll}
\hline Parameter Path & Estimate \\
\hline
\end{tabular}

\section{Training effect on mediator}

$\begin{array}{lll}\text { a11 } & \text { active }->\text { bias1 } & -0.396[-0.697,-0.099]^{*} \\ \text { a12 } & \text { active }->\text { bias2 } & 0.168[-0.168,0.507] \\ \text { a13 } & \text { active }->\text { bias3 } & -0.163[-0.488,0.259] \\ \text { a14 } & \text { active }->\text { bias4 } & -0.136[-0.466,0.185] \\ \text { a21 } & \text { sham }->\text { bias1 } & 0[0,0] \\ \text { a22 } & \text { sham }->\text { bias2 } & 0[0,0] \\ \text { a23 } & \text { sham }->\text { bias3 } & 0[0,0] \\ \text { a24 } & \text { sham }->\text { bias4 } & 0[0,0]\end{array}$

\section{Mediator effect on outcome}

b $\quad \operatorname{bias}(\mathrm{t})->\operatorname{dep}(\mathrm{t}) \quad 0.244[0.123,0.444]^{*}$

\section{Training effect on outcome}

$\begin{array}{lll}\text { c'11 } & \text { active }->\text { dep1 } & -0.05[-0.311,0.196] \\ \text { c'12 } & \text { active }->\text { dep2 } & -0.312[-0.593,0.037] \\ \text { c'13 } & \text { active }->\text { dep3 } & 0.182[-0.149,0.536] \\ \text { c'14 } & \text { active }->\text { dep4 } & -0.338[-0.642,-0.037]^{*} \\ \text { c'21 } & \text { sham }->\text { dep1 } & -0.215[-0.464,0.029] \\ \text { c'22 } & \text { sham }->\text { dep2 } & -0.031[-0.34,0.276] \\ \text { c'23 } & \text { sham }->\text { dep3 } & 0.012[-0.306,0.363] \\ \text { c'24 } & \text { sham }->\text { dep4 } & -0.145[-0.446,0.139]\end{array}$

\section{Autoregressive effect}

$\begin{array}{lll}\mathrm{m} & \operatorname{bias}(\mathrm{t}-1)->\operatorname{bias}(\mathrm{t}) & 0.701[0.472,0.937]^{*} \\ \mathrm{y} & \operatorname{dep}(\mathrm{t}-1)->\operatorname{dep}(\mathrm{t}) & 0.852[0.621,0.988]^{*}\end{array}$

a Sham ABMT effects on attentional bias were fixed to 0. Models in which these effects are freely estimated can be found in the supplemental materials. 


\section{Table 2}

\section{Effect decomposition for constrained contemporaneous mediation model}

\begin{tabular}{|c|c|c|c|}
\hline Paths/effects for final time point & Parameters & Active & Sham \\
\hline \multicolumn{4}{|l|}{ Indirect Effects } \\
\hline train $->$ bias $1->$ dep $1->$ dep $2->$ dep $3->$ dep 4 & $\mathrm{a}(1 \mid 2) 1 \times \mathrm{b} \times \mathrm{y}^{\wedge} 3$ & $-0.06[-0.135,-0.019]^{*}$ & $0[0,0]$ \\
\hline train $->$ bias $1->$ bias2 $->$ dep2 $->$ dep3 $->$ dep4 & $\mathrm{a}(1 \mid 2) 1 \times \mathrm{m} \times \mathrm{b} \times \mathrm{y}^{\wedge} 2$ & $-0.049[-0.116,-0.016]^{*}$ & $0[0,0]$ \\
\hline train $->$ bias1 $->$ bias2 $->$ bias $3->$ dep 3 -> dep4 & $\mathrm{a}(1 \mid 2) 1 \times \mathrm{m}^{\wedge} 2 \times \mathrm{b} \times \mathrm{y}$ & $-0.041[-0.125,-0.011]^{*}$ & $0[0,0]$ \\
\hline train $->$ bias $1->$ bias $2->$ bias $3->$ bias $4->$ dep 4 & $\mathrm{a}(1 \mid 2) 1 \times \widehat{\mathrm{m} \widehat{3}} \times \mathrm{b}$ & $-0.033[-0.235,-0.007]^{*}$ & $0[0,0]$ \\
\hline train -> bias2 -> dep2 -> dep3 -> dep4 & $\mathrm{a}(1 \mid 2) 2 \times \mathrm{b} \times \mathrm{y}^{\wedge} 2$ & $0.03[-0.026,0.106]$ & $0[0,0]$ \\
\hline train -> bias2 -> bias3 -> dep3 -> dep4 & $\mathrm{a}(1 \mid 2) 2 \times \mathrm{m} \times \mathrm{b} \times \mathrm{y}$ & $0.024[-0.016,0.106]$ & $0[0,0]$ \\
\hline train -> bias2 -> bias3 $->$ bias4 -> dep4 & $\mathrm{a}(1 \mid 2) 2 \times \mathrm{m}^{\wedge} 2 \times \mathrm{b}$ & $0.02[-0.013,0.125]$ & $0[0,0]$ \\
\hline train $->$ bias $3->$ dep3 $->$ dep 4 & $\mathrm{a}(1 \mid 2) 3 \times \mathrm{b} \times \mathrm{y}$ & $-0.034[-0.118,0.06]$ & $0[0,0]$ \\
\hline train $->$ bias 3 -> bias $4->$ dep 4 & $\mathrm{a}(1 \mid 2) 3 \times \mathrm{m} \times \mathrm{b}$ & $-0.028[-0.103,0.043]$ & $0[0,0]$ \\
\hline train $->$ bias 4 -> dep4 & $\mathrm{a}(1 \mid 2) 4 \times \mathrm{b}$ & $-0.033[-0.133,0.05]$ & $0[0,0]$ \\
\hline overall indirect effect & & $-0.204[-0.407,-0.039]^{*}$ & $0[0,0]$ \\
\hline \multicolumn{4}{|l|}{ Direct Effects } \\
\hline train -> dep1 -> dep2 -> dep3 -> dep4 & $\mathrm{c}^{\prime}(1 \mid 2) 1 \times \mathrm{y}^{\wedge} 3$ & $-0.031[-0.223,0.104]$ & $-0.133[-0.35,-0.006]^{*}$ \\
\hline train $->$ dep $2->$ dep $3->$ dep 4 & $\mathrm{c}^{\prime}(1 \mid 2) 2 \times \mathrm{y}^{-2}$ & $-0.227[-0.506,0.002]$ & $-0.023[-0.272,0.175]$ \\
\hline train $->$ dep3 $->$ dep 4 & $c^{\prime}(1 \mid 2) 3 \times y$ & $0.155[-0.107,0.494]$ & $0.011[-0.239,0.329]$ \\
\hline train -> dep4 & $c^{\prime}(1 \mid 2) 4$ & $-0.338[-0.642,-0.037]^{*}$ & $-0.145[-0.446,0.139]$ \\
\hline overall direct effect & & $-0.44[-0.774,-0.092]^{*}$ & $-0.29[-0.62,-0.032]^{*}$ \\
\hline Total Effect & & $-0.644[-0.924,-0.327]^{*}$ & $-0.29[-0.62,-0.032]^{*}$ \\
\hline
\end{tabular}

${ }^{a}$ Sham ABMT effects on attentional bias were fixed to 0. Models in which these effects are freely estimated can be found in the supplemental materials. 


\section{Table 3}

SEM paths for latent growth model

\begin{tabular}{ll}
\hline Parameter Path & Estimate \\
\hline
\end{tabular}

Training effect on mediator

$\begin{array}{lll}\text { a1 } & \text { active }->\text { bias slope } & -0.073[-0.156,-0.006]^{*} \\ \text { a2 } & \text { sham }->\text { bias slope } & 0[0,0]\end{array}$

Mediator effect on outcome

b bias slope -> dep slope $1.898[0.409,5.433]^{*}$

\section{Training effect on outcome}

c'1 active $->$ dep slope $\quad 0[-0.06,0]$

c'2 $\quad$ sham -> dep slope $\quad-0.057[-0.136,0]$

Note:

$*$ indicates $\mathrm{p}<.05$ 
Table 4

Effect decomposition for the bounded latent growth model

\begin{tabular}{ll}
\hline Active & Sham \\
\hline
\end{tabular}

\section{Indirect Effects}

$$
-0.554[-0.929,-0.174]^{*} \quad 0[0,0]
$$

\section{Direct Effects}

$$
0[-0.239,0] \quad-0.229[-0.544,0]
$$

\section{Total Effects}

\begin{tabular}{l}
$-0.554[-0.824,-0.218]^{*}-0.229[-0.544,0]$ \\
\hline Note: \\
$*$ indicates $\mathrm{p}<.05$
\end{tabular}




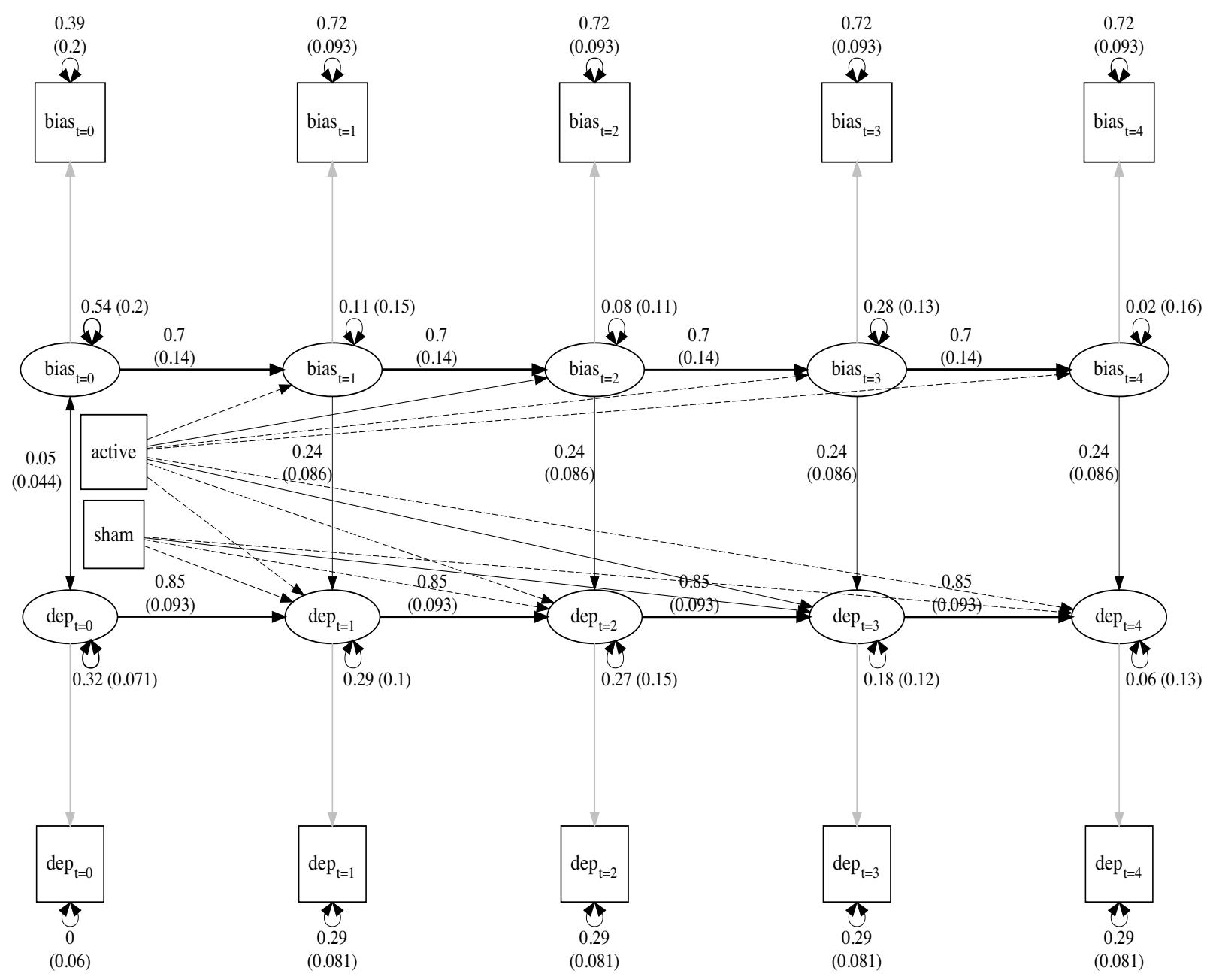

\section{Figure 1}

Contemporaneous constrained mediation model. Path coefficients and standard errors are not shown for ABMT effects for parsimony but the path widths are proportional to the size of coefficient. Dotted lines indicate negative relationships and solid lines indicate positive relationships. Grey lines indicate path coefficient were fixed to 1. The path coefficients for the training effects can be found in Table 1. 


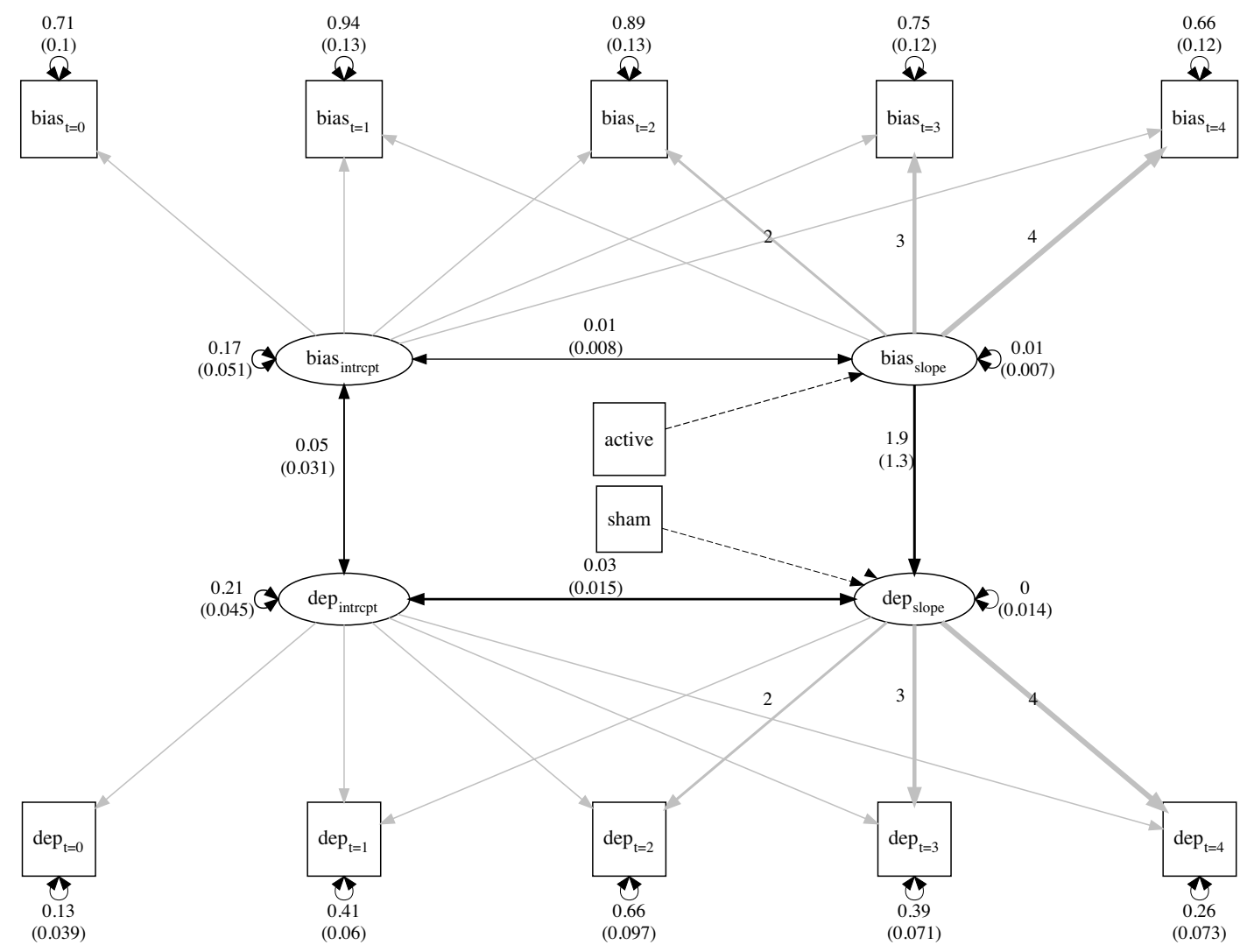

\section{Figure 2}

Latent growth model. Path widths are proportional to the size of coefficient. Dotted lines indicate negative relationships and solid lines indicate positive relationships. Grey lines indicate path coefficient were fixed to 1 unless otherwise noted. 